of dupilumab treatment, consistent with results in adults and adolescents. These data suggest that no routine laboratory monitoring for hematology/chemistry parameters is required in adolescents with $\mathrm{AD}$ prior to or during dupilumab treatment.

\section{CHARACTERIZATION OF PEDIATRIC PATIENTS UNDER ALLERGEN-SPECIFIC IMMUNOTHERAPY AT A LEVEL II HOSPITAL}

Catarina M Francisco*, JF Ribeiro, A Monteiro, P Guerra, RS Oliveira. Sousa Martins Hospital

10.1136/archdischild-2021-europaediatrics. 162

Allergen-Specific Immunotherapy (ASI) is being used on the treatment of respiratory allergies for over a century, aiming to reduce individual hypersensitivity to specific allergens, and is proven to be very effective on the long term.

Goal Evaluation of the patients followed in the Paediatric Allergology clinic at a level II Hospital treated with ASI.

Retrospective and descriptive study of the efficiency of ASI in patients attending our Allergology inpatient clinic between 2016 and 2020.

The sample was characterized according to patients' gender, age, initial diagnosis, method of allergy testing (blood IgE or Skin Prick Testing) and the type of ASI used.

During the estimated period, a total of 44 patients were treated with ASI.

Of these, $27(61 \%)$ are male, with a median age of 11 years old on the first day of treatment (minimum age: 6 years old, maximum age: 17 years old).

As to the diagnosis: 34 (77\%) have Allergic Rhinitis, 20 (46\%) have Allergic Asthma, 9(21\%) Allergic Conjuntctivitis, 3 (7\%) Urticaria, and 2 (5\%) Atopic Dermathitis. The majority of patients have more than one condition identified.

All the patients underwent Skin Prick Testing and 60\% also did blood tests.

Grasses and domestical mites were the agents more frequently identified as inducers of Hypersensitivy, and 36\% of the patients received monotherapy.

Subcutaneous admnistrations was the prefered method in 2/ 3 of the patients.

There were no adverse effects observed until today and the ASI was proven to be effective to reduce allergic symptomatology.

ASI is the only treatment modality known to effectively change the natural course of Allergic disease.

However, it is essential to thoroughly select the patients that can qualify for the treatment, as to the specific allergen extracts being used, so that we can optimize therapy benefits.

\section{AMOXICILLIN ALLERGY IN CHILDREN... COMMON OR UNCOMMON? (\#151 WITH CHANGES)}

Íris Santos Silva*, Catarina Macedo Francisco, Joana Filipe Ribeiro, João Virtuoso, Pedro Guerra, Rita S Oliveira. ULS Guarda

\subsection{6/archdischild-2021-europaediatrics. 163}

$\beta$-Lactam antibiotics are safe and cost-effective antibiotics, being amoxicillin the most common antibiotic used among the paediatric population.
Many patients report allergic reactions to this antibiotic, but amoxicillin allergy range between 1-10\%. However, clinicians hesitate to prescribe it when a suspected, but unproven, allergy exists. Our aim is to confirm amoxicillin allergy in children with clinical suspicion.

This study was done between January 2018 and December 2020, in children younger than 18 years, admitted to the emergency room with suspicion of clinical allergic reaction to amoxicillin. According to the protocol of our hospital, they were referred for pediatric allergology appointment to perform prick tests and afterwards oral provocation test.

A total of 57 cases were referred for evaluation. The average age was 8.4

(1-17) years old, and 53\% were female. The suspicion was based on late rash reaction presented in $68, .4 \%$, urticarial exanthema in $19.3 \%$, edema in $8.8 \%$, vomit in $8.8 \%$, cutaneous rash in $7 \%$ and dyspnea in 2,1\%. In 18 patients, specific IgE screening for amoxicillin was performed, but all results were negative. All children did a prick test for amoxicillin and oral provocation test. There were no positive results for prick tests, but two positive results in the oral provocation test $(3.5 \%)$.

Confirmation of amoxicillin allergy, before deciding to use it is or not, is an important tool for antimicrobial stewardship and, consequently, to decrease the rate of antibiotic resistance. So far, in our hospital, there were only two positive results.

\section{SERUM PERIOSTIN AS A POTENTIAL BIOMARKER FOR ASTHMA SYMPTOMS IN CHILDREN WITH HISTORY OF RESPIRATORY SYNCYTIAL VIRUS INFECTION}

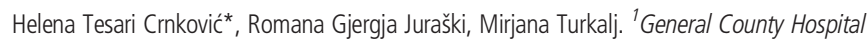
Požega; ${ }^{2}$ Faculty of Medicine, J.J. Strossmayer University of Osijek

\subsection{6/archdischild-2021-europaediatrics. 164}

Periostin is a matricellular protein upregulated in response to IL-4 and IL-13, that have a role in development of allergic diseases. Previous studies reported that periostin can be a noninvasive biomarker of $\mathrm{T} 2$-driven inflammatory response in asthma in adults, with inconsistent results in children. None of the studies examined the association of serum periostin levels with asthma symptoms in children who have been infected with respiratory syncytial virus (RSV) in the first two years of life. The aim of this study was to determine the usefulness of serum periostin levels as a potential biomarker for asthma, especially recent asthma symptoms in children.

This prospective study observed 72 children from birth. RSV infection was confirmed with positive serum specific RSV Immunoglobulin $\mathrm{G}$ (IgG) at one and/or two years of age. Asthma was diagnosed according to International Study of Asthma and Allergies in Childhood (ISAAC) questionnaire at 10 years of age. Fractional exhaled nitric oxide (FeNO), lung function, skin prick test and blood samples for analysis of specific immunoglobulin $\mathrm{E}$ (sIgE) on standard pallet of inhalant allergens, total $\operatorname{IgE}(\mathrm{t} \operatorname{IgE})$ and periostin were provided.

At 10 years of age, asthma was diagnosed in 23 (31.9\%) of the observed children. In 15 (20.8\%) of them who reported asthma symptoms during the last

12 months, median serum periostin levels were $40.04 \mathrm{ng} /$ ml. In $57(79.2 \%)$ children who were free of asthma symptoms in the last 12 months, mean serum periostin levels were $30.57 \mathrm{ng} / \mathrm{ml}$. Serum periostin levels correlated significantly 
with recent asthma symptoms (Kendall's Tau-B $=0.236, \mathrm{p}=$ 0.016).

Serum periostin levels significantly correlated with asthma symptoms during the last 12 months in 10-year-old children who were infected with RSV during their first two years of life. Our preliminary results suggest that serum periostin level may be useful in diagnosing asthma in children, especially those with recent asthma symptoms and history of RSV infection in infancy.

\section{ORAL BETA-LACTAM CHALLENGE TEST WITHOUT ANTECEDENT SKIN TESTING IN CHILDREN WITH HISTORY OF ALLERGY TO BETA-LACTAM}

Iva Mihatov Štefanović* Renata Vrsalović, Gabrijela Perić Marković. University Hospital Center Sestre Milosrdnice

\subsection{6/archdischild-2021-europaediatrics. 165}

Beta-lactam (BL) antibiotics are among the most prescribed drugs globally but can provoke hypersensitivity reactions which can lead to incorrectly labelling as 'allergy'. Data on prevalence and incidence of drug hypersensitivity reactions (DHRs) are limited, especially in the pediatric age. It is important to assess subjects with history of hypersensitivity reactions to $\mathrm{BL}$ as up to $70 \%$ are not allergic based on diagnostic test results. Laboratory tests for identifying children who are allergic to drugs have low diagnostic accuracy and predictive value. The gold standard to diagnose DHR is drug provocation test (DPT). DHRs are classified as immediate or nonimmediate/delayed reactions. Mild delayed cutaneous reactions including maculopapular rashes and urticaria/angioedema are common reactions in children and often occur in the setting of viral infections. In mild cutaneous delayed reactions, some clinicians suggest performing only DPT because of its high negative predictive value, without skin testing and serum IgE measurement while other suggest SPT and/or specific IgE to culprit drug if patient/caregiver cannot provide a detailed description of the previous reaction.

Aim: to investigate the proportion of positive oral provocation tests in children labeled as BL antibiotic allergy.

Direct oral challenge was performed in patients with history of benign rash associated with beta-lactam antibiotic. Because detailed description of suspected allergic reaction could not be provided from all caregivers, all patients went specific $\operatorname{IgE}$ to amoxicillin and/or basophil degranulation test to culprit drug which was negative. 19 patients were included, average age 6 years and 1 month. In 17 patients suspected allergic reaction was caused by amoxicillin (AMX); 8 of which in combination with clavulanic acid, one with phenoxymethylpenicillin and one with cephalexin. A DPT involved open 3step graded oral provocation test with AMX, time interval between the doses was $2 \mathrm{~h}$. The patients were monitored for $24 \mathrm{~h}$ after challenge and were discharged with instructions to call in the event of a delayed reaction.

After DPT there was no reaction in $89 \%$ of patients, one patient developed benign rash and one drug-induced enterocolitis syndrome.

BL antibiotics can be safely readministered to children with history of benign rash to beta-lactams using graded oral challenge in medically supervised settings if $\operatorname{spIgE}$ is negative even if uncertain history. It is important to establish a correct diagnosis of BL antibiotic allergy since using alternative non- $\beta$-lactam antibiotics in these patients leads to higher healthcare costs and adverse events.

\section{EFFICACY OF DUPILUMAB IN CHILDREN WITH UNCONTROLLED, MODERATE-TO-SEVERE TYPE 2 ASTHMA, WITH AND WITHOUT EVIDENCE OF ALLERGY ENROLLED IN PHASE 3 VOYAGE}

Stanley J Szefler, Leonard B Bacharier, Jorge F Maspero, Nikos Papadopoulos, Christian Domingo*, Nadia Daizadeh, David J Lederer, Megan Hardin, Juby A Jacob-Nara, Yamo Deniz, Rebecca Gall, Benjamin Ortiz, Michel Djandji, Paul J Rowe. Royal Manchester Children's Hospital, Manchester, UK; 2nd Pediatric Clinic, University of Athens, Greece

\subsection{6/archdischild-2021-europaediatrics. 166}

The allergic phenotype is an important subset of asthma patients with type 2 inflammation. Dupilumab, a fully human monoclonal antibody, blocks the shared receptor component for IL-4/IL-13, key and central drivers of type 2 inflammation in multiple diseases. In phase 3 VOYAGE, add-on dupilumab $100 \mathrm{mg} / 200 \mathrm{mg}$ (body weight $\leq 30 \mathrm{~kg} />30 \mathrm{~kg}$, respectively) every 2 weeks vs placebo, reduced severe asthma exacerbations by $59.3 \% \quad(\mathrm{P}<0.0001)$ and improved lung function in children aged 6 to $<12$ years with uncontrolled moderate-to-severe type 2 asthma (baseline blood eosinophils $\geq 150$ cells $/ \mu \mathrm{l}$ or FeNO $\geq 20 \mathrm{ppb}$ ). This analysis evaluated the efficacy of dupilumab in pediatric patients with type 2 asthma with/without evidence of allergic asthma (total serum $\operatorname{IgE} \geq 30 \mathrm{IU} / \mathrm{mL}$ and $\geq 1$ perennial aeroallergen-specific $\mathrm{IgE} \geq 0.35 \mathrm{kU} / \mathrm{L}$ at baseline).

Annualized severe exacerbation rate during the 52-week treatment period was assessed using a negative binomial model.

350 pediatric patients with type 2 asthma were enrolled: of which, 261 had evidence of allergic asthma and 89 did not. Baseline characteristics were similar between subgroups, except for prevalence of ongoing atopic comorbidities $(99.6 \%$ vs $77.5 \%$ ) and median levels of type 2 biomarkers (blood eosinophils: 540.00 cells $/ \mu \mathrm{L}$ vs 310.00 cells $/ \mu \mathrm{L} ;$ FeNO: $28 \mathrm{ppb}$ vs $13 \mathrm{ppb}$; total serum IgE: $657.00 \mathrm{IU} / \mathrm{mL}$ vs $107.00 \mathrm{IU} / \mathrm{mL}$ ) which were all higher in patients with vs without evidence of allergic asthma. Dupilumab vs placebo significantly reduced annualized severe exacerbation rate by $62 \%(\mathrm{P}<0.0001)$ in patients with, and $51 \%(\mathrm{P}<0.05)$ in patients without evidence of allergic asthma. No significant interaction was observed between the treatment effect and evidence of allergic asthma. In the overall safety population, the incidence of treatment-emergent adverse events (TEAEs) was similar across treatment groups; the most common TEAE occurring more frequently in the dupilumab group was injection site erythema (12.9\% dupilumab vs $9.7 \%$ placebo).

The majority of pediatric type 2 asthma patients enrolled in VOYAGE had evidence of allergic asthma; these patients had very high levels of type 2 biomarkers. Dupilumab demonstrated efficacy in reducing severe asthma exacerbations in children aged 6 to $<12$ years with uncontrolled, moderate-tosevere type 2 asthma, with or without evidence of allergic asthma. 